\title{
UNIVERSITYOF
}

FORWARD

THINKING

WESTMINSTER用

WestminsterResearch

http://www.westminster.ac.uk/westminsterresearch

\section{Salivary diurnal cortisol profiles in patients suffering from chronic breathlessness receiving supportive and palliative care services: a cross-sectional study \\ Ryan, R., Clow, A., Spathis, A., Smyth, N., Barclay, S., Fallon, M. and Booth, S.}

NOTICE: this is the authors' version of a work that was accepted for publication in Journal of Pharmaceutical Sciences. Changes resulting from the publishing process, such as peer review, editing, corrections, structural formatting, and other quality control mechanisms may not be reflected in this document. Changes may have been made to this work since it was submitted for publication. A definitive version was subsequently published in Psychoneuroendocrinology, doi: 10.1016/j.psyneuen.2017.01.025

The final definitive version in Psychoneuroendocrinology will be available online at:

https://dx.doi.org/10.1016/j.psyneuen.2017.01.025

(C) 2017. This manuscript version is made available under the CC-BY-NC-ND 4.0 license http://creativecommons.org/licenses/by-nc-nd/4.0/

The WestminsterResearch online digital archive at the University of Westminster aims to make the research output of the University available to a wider audience. Copyright and Moral Rights remain with the authors and/or copyright owners.

Whilst further distribution of specific materials from within this archive is forbidden, you may freely distribute the URL of WestminsterResearch: ((http://westminsterresearch.wmin.ac.uk/)).

In case of abuse or copyright appearing without permission e-mail repository@westminster.ac.uk 



\section{Accepted Manuscript}

Title: Salivary diurnal cortisol profiles in patients suffering from chronic breathlessness receiving supportive and palliative care services: a cross-sectional study

Authors: Richella Ryan, Angela Clow, Anna Spathis, Nina Smyth, Stephen Barclay, Marie Fallon, Sara Booth

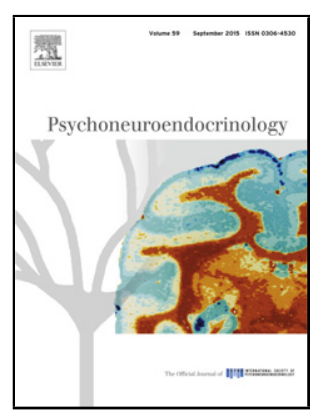

PII: S0306-4530(16)30785-5

DOI: http://dx.doi.org/doi:10.1016/j.psyneuen.2017.01.025

Reference: PNEC 3522

To appear in:

Received date: $\quad$ 19-10-2016

Revised date: $\quad 16-12-2016$

Accepted date: $\quad$ 24-1-2017

Please cite this article as: Ryan, Richella, Clow, Angela, Spathis, Anna, Smyth, Nina, Barclay, Stephen, Fallon, Marie, Booth, Sara, Salivary diurnal cortisol profiles in patients suffering from chronic breathlessness receiving supportive and palliative care services: a cross-sectional study.Psychoneuroendocrinology http://dx.doi.org/10.1016/j.psyneuen.2017.01.025

This is a PDF file of an unedited manuscript that has been accepted for publication. As a service to our customers we are providing this early version of the manuscript. The manuscript will undergo copyediting, typesetting, and review of the resulting proof before it is published in its final form. Please note that during the production process errors may be discovered which could affect the content, and all legal disclaimers that apply to the journal pertain. 
Title:

Salivary diurnal cortisol profiles in patients suffering from chronic breathlessness receiving supportive and palliative care services: a cross-sectional study

\section{Corresponding author ${ }^{1}$ :}

Dr. Richella Ryan,

Email: rcr41@medschl.cam.ac.uk

Phone: 0044 (0)7570 896978

\section{Other authors:}

Prof. Angela Clow ${ }^{\mathrm{c}}$

Dr. Anna Spathis ${ }^{a, b}$

Dr. Nina Smyth ${ }^{c}$

Dr. Stephen Barclay ${ }^{b}$

Prof. Marie Fallon ${ }^{\mathrm{d}}$

Dr. Sara Booth ${ }^{a}$

\section{Affiliations:}

${ }^{a}$ Cambridge University Hospitals NHS Foundation Trust,

Palliative Care Department,

Addenbrooke's Hospital,

Hill's Road,

Cambridge,

CB2 0QQ

${ }^{\mathrm{b}}$ Primary Care Unit,

Department of Public Health and Primary Care,

University of Cambridge,

Institute of Public Health,

University Forvie Site,

Robinson Way,

Cambridge,

CB2 OSR

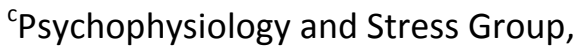

University of Westminster,

Department of Psychology,

115 New Cavendish Street,

London,

W1W 6UW

${ }^{d}$ Edinburgh Cancer Research Centre (IGMM),

The University of Edinburgh,

Crewe Road South,

Edinburgh,

EH4 2XR

\footnotetext{
${ }^{1}$ Present address $=\mathrm{b}$
} 


\section{Highlights}

- Flatter mean diurnal cortisol slope in patients compared to healthy controls

- Differences in mean diurnal cortisol slope depending on severity of breathlessness

- Positive correlation for diurnal cortisol slope and MRC dyspnoea grade in patients

- Aberrant cortisol profile patterns in patients with moderate-to-severe breathlessness 


\section{Abstract}

Chronic breathlessness is a common source of psychological and physical stress in patients with advanced or progressive disease, suggesting that hypothalamic-pituitary-adrenal (HPA) axis dysregulation may be prevalent. The aim of this study was to measure the salivary diurnal cortisol profile in patients receiving supportive and palliative care for a range of malignant and nonmalignant conditions and to compare the profile of those experiencing moderate-to-severe disability due to breathlessness against that of patients with mild/no breathlessness and that of healthy controls. Saliva samples were collected over two consecutive weekdays at 3, 6, and 12 hours after awakening in 49 patients with moderate-to-severe breathlessness [Medical Research Council (MRC) dyspnoea grade $\geq 3$ ], 11 patients with mild/no breathlessness (MRC dyspnoea grade $\leq 2$ ), and 50 healthy controls. Measures of breathlessness, stress, anxiety, depression, wellbeing and sleep were examined concomitantly. The diurnal cortisol slope (DCS) was calculated for each participant by regressing log-transformed cortisol values against collection time. Mean DCS was compared across groups using ANCOVA. Individual slopes were categorised into one of four categories: consistent declining, consistent flat, consistent ascending and inconsistent. Controlling for age, gender and socioeconomic status, the mean DCS was significantly flatter in patients with moderate-to-severe breathlessness compared to patients with mild/no breathlessness and healthy controls $[F(2,103)$ $=45.64, p<0.001]$. Furthermore, there was a higher prevalence of flat and ascending cortisol profiles in patients with moderate-to-severe breathlessness $(23.4 \%)$ compared to healthy controls ( $0 \%)$. The only variable which correlated significantly with DCS was MRC dyspnoea grade $(r s=0.29, p<0.05)$. These findings suggest that patients with moderate-to-severe breathlessness have evidence of HPA axis dysregulation and that this dysregulation may be related to the functional disability imposed by breathlessness. 
Richella Ryan

\section{Keywords}

'cortisol', 'hypothalamic-pituitary-adrenal axis', 'breathlessness', 'palliative care', 'dyspnoea', 'saliva' 


\section{Salivary diurnal cortisol profiles in patients suffering from chronic breathlessness receiving supportive and palliative care services: a cross- sectional study}

\section{Introduction}

Chronic breathlessness is a common symptom of advanced cardiorespiratory disease, affecting $>90 \%$ of people with advanced COPD, $>60 \%$ of people with advanced heart disease and $10-70 \%$ of people with advanced cancer (Solano et al., 2006). It is a considerable source of psychosocial and physical stress, being associated with fear, anxiety, social isolation and functional disability (Booth et al., 2003). It has also been shown to predict mortality, independent of lung function, in a range of conditions, including COPD (Nishimura et al., 2002), pulmonary fibrosis (Nishiyama et al., 2010), and older adulthood (Ahmed et al., 2012). These effects suggest a potential role for the stress hormone system in the experience of breathlessness.

The hypothalamic-pituitary-adrenal (HPA) axis is a major regulator of the physiological stress response, exerting its effects through its end-hormone, cortisol. Basal cortisol secretion has a marked circadian rhythm, characterised by a rapid rise in cortisol secretion upon awakening, followed by a fall in cortisol levels throughout the day (Smyth et al., 2013). This pattern becomes disrupted in the context of prolonged or repeated exposure to stressful triggers, often manifesting as loss or blunting of diurnal variation in cortisol secretion (Miller et al., 2007). Such dysregulation has been observed in a wide range of chronic diseases, both malignant (Abercrombie et al., 2004) and non-malignant (Hackett et al., 2014), and has been associated with negative disease outcomes and characteristics. Flatter diurnal rhythms have been shown to predict mortality secondary to cancer (Sephton et al., 2000; Cohen et al., 2012; Sephton et al., 2013) and cardiovascular disease (Kumari et al., 2011 ). They have also been associated with more advanced cancer stages (Sephton et 
al., 2000; Sephton et al., 2013), greater functional disability in cancer (Weinrib et al., 2011; Sephton et al., 2013) and worse physical performance in older adulthood (Gardner et al., 2013).

To date, the relationship of the HPA axis with the physical symptomatology of chronic progressive diseases has been explored to a limited degree only. A few studies have described diurnal cortisol profiles in relation to fatigue in patients with cancer, but findings have been inconsistent (Sephton et al., 2000; Weinrib et al., 2001; Bower et al., 2005, Sephton et al., 2013). One study has identified a relationship between HPA axis function and the cancer symptom cluster of pain, fatigue and depression, but it did not assess diurnal cortisol rhythmicity (Thornton et al., 2010). Symptoms are increasingly understood in the literature to be psychoneurological responses to disease, driven by pathophysiological mechanisms which are distinct from, though related to, the underlying associated disease (Miller et al., 2008; Dantzer et al. 2012; Kim et al. 2012). It is, thus, important to identify these pathophysiological mechanisms to allow therapeutic targeting of symptoms independent of disease.

No studies have examined the relationship between chronic breathlessness and HPA axis function. The literature points to a number of specific factors which are suggestive of a link, however. First, there is a well-established relationship between breathlessness and anxiety (von Leupoldt et al., 2011), which might promote a chronic stress response. Second, the perception of breathlessness is processed in the same parts of the cortico-limbic system (insula, amygdala and anterior cingulate cortex) as other aversive sensations such as pain, hunger and thirst (von Leupoldt et al., 2009), all of which are known to activate the stress response. Finally, the aforementioned independent relationship between breathlessness severity and mortality, across a range of contexts, is currently unexplained; HPA axis dysregulation, in the context of breathlessness, represents one possible explanation needing exploration. 


\subsection{Hypothesis, aims and objectives}

We hypothesised that patients suffering from chronic breathlessness due to chronic progressive disease would have evidence of HPA axis dysregulation, represented by a flatter diurnal cortisol slope. To investigate this, we aimed to measure the salivary diurnal cortisol profile in patients under the care of supportive and palliative care services, who were experiencing moderate-to-severe disability due to breathlessness, comparing this profile against that of non-breathless patients and that of healthy adults. Specific objectives were:

1) To compare the mean diurnal cortisol slope (slope of the decline in cortisol across the day) in patients with moderate to severe breathlessness (Medical Research Council, MRC $\geq 3$ ), against that of non-breathless patients (MRC $\leq 2$ ) and that of healthy adults.

2) To compare pre-defined diurnal cortisol slope categories between patients with moderateto-severe breathlessness and healthy adults.

\section{Material and Methods}

Study procedures for patient participants were approved by the National Research Ethics Service Committee, East of England-Norfolk (13/EE/0021). It was part of a larger programme of research, registered on the ISRCTN registry (ISRCTN70836126). Study procedures for the healthy control group were approved by the ethics committee of the University of Westminster. Written informed consent was obtained from all participants prior to enrolment.

\subsection{Participants- patient group (MRC $\geq 3$ and $M R C \leq 2$ subgroups)}

Patients were recruited from a hospital and a hospice in the Cambridgeshire region of the UK. Patients from the hospital were recruited via a specialist breathlessness service (Breathlessness Intervention Service, BIS), a service delivered by hospital-based personnel to patients living with chronic breathlessness in the community. They were considered eligible for the study if they met the 
referral criteria for BIS: 1) had a diagnosed or investigated cause for breathlessness, 2) were troubled by breathlessness despite optimal medical therapy, and 3) might benefit from a selfmanagement programme. Patients from the hospice were recruited via the day therapy, community, and supportive care services, irrespective of whether or not they were breathless. Patients were excluded from the study if they: 1) had received systemic steroids within the preceding 4 weeks, 2) had asthma or a non-physical cause of breathlessness, 3) were unable to provide informed consent, 4) were currently receiving inpatient hospital or hospice care, or 5) had rapidly progressive disease such that they were imminently dying.

This recruitment strategy ensured that most of the patients would have advanced disease, that a large proportion would be suffering from moderate to severe breathlessness (MRC $\geq 3$ ) and that a subsample would not be suffering from breathlessness (MRC $\leq 2$ ).

\subsection{Participants- healthy control group}

The data of participants recruited to a separate study, conducted at the University of Westminster (London) (Evans et al., 2007), were used to provide a healthy control group, against which the patient group was compared. This group consisted of community-dwelling healthy older adults recruited in the South-West London area. Volunteers were excluded from this study if they: 1) were receiving steroid medication, 2) had an acute or chronic medical condition, or 3) had evidence of cognitive impairment based on the Mini-Mental State Examination.

\subsection{General Procedures}

Study procedures were the same for the patient and healthy control groups, unless stated otherwise.

Demographic and clinical details were obtained through a structured interview, followed by review of patients' clinical records where necessary. All participants were instructed in the study procedures and provided with the following study materials: 1) a saliva collection pack consisting of pre-labelled 
Cortisol Salivette ${ }^{\circledR}$ devices (Sarstedt, Ltd., Leicester), 2) full-written instructions on how to use the collection devices, 3) self-recording sheets for documenting the timing of saliva collections, and 4) self-administered questionnaires. Upon completion of the study, materials were returned either by collection from participants' homes, by post or upon attendance at a hospice or hospital clinic.

\subsection{Measures}

Salivary diurnal cortisol and demographic variables were assessed for both patients and healthy controls. All other measures were obtained for patients only. Questionnaires measuring breathlessness, psychological status and sleep were self-administered unless otherwise stated.

\subsubsection{Salivary diurnal cortisol assessment}

Participants were instructed to complete a two-day saliva collection protocol at home, on two consecutive weekdays during which nothing out of the ordinary was expected to happen. They provided 3 samples per day, synchronised to the time of awakening: 3, 6, and 12 hours after awakening.

Cortisol Salivette ${ }^{\circledast}$ devices consist of a small synthetic swab contained in a sterile plastic centrifugation tube. Each plastic tube was pre-labelled with a different instruction about the collection day and time relative to awakening (e.g. 'day 1, 3 hours'; 'day 2, 6 hours'). Participants were instructed to chew on the swab for at least 1 minute and then return it to the tube. They were asked to record the time that each sample was collected on a self-recording sheet and were instructed to avoid eating, drinking, smoking or brushing their teeth for 30 minutes prior to each sample. To avoid mould formation in the Salivette, participants were advised to store their samples in their home freezer at the end of each day until postage or collection. At the laboratory, samples were stored at $-20^{\circ} \mathrm{C}$ until analysis.

All samples were analysed at the University of Westminster using their standard laboratory protocols. Cortisol concentration was determined by Enzyme Linked Immunosorbent Assay (ELISA) 
developed by Salimetrics LLC (USA), which has the following assay characteristics: standard range $=0.33-82.77 \mathrm{nmol} / \mathrm{l}$, assay sensitivity $=0.19 \mathrm{nmol} / \mathrm{I}$ (lower limit of detection), correlation with serum cortisol=0.91 ( $p<0.0001, n=47$ samples). After centrifuging thawed samples at 3500rpm for 10 minutes, duplicate analysis of samples was undertaken. The intra-assay coefficient of variation was $<10 \%$ for all samples.

The diurnal cortisol slope (DCS) was calculated for each individual in order to summarise the diurnal cortisol profile using the 3-, 6- and 12-hour post-awakening samples. It was first calculated for each separate day of collection. After confirming lack of a significant day-difference, an aggregated slope across the two days was subsequently calculated by regressing log-transformed cortisol values on sample collection time across the two days (6 samples). Using this approach, the slope of the linear regression line (unstandardized beta coefficient) represents the diurnal cortisol rhythm (Smyth et al., 1997; Sephton et al., 2000).

\subsubsection{Demographic and clinical measures}

Demographic data including age, gender and subjective socio-economic status (Adler et al., 2000) were recorded for all participants. The following clinical information was obtained for the patient group: primary diagnosis, co-morbidities, medication, current use of steroid inhalers, Karnofsky Performance Status (Karnofsky and Burchenal, 1949), and Charlson Co-Morbidity Index (Charlson et al., 1987).

\subsubsection{Measures of breathlessness}

In view of its multi-dimensional nature, three different measures were used to assess breathlessness, each measuring a different dimension.

\subsubsection{Medical Research Council (MRC) Dyspnoea Scale}

Perceived functional disability as a result of breathlessness was measured using the Medical Research Council (MRC) dyspnoea scale (Fletcher et al., 1959), used with the permission of the 
Medical Research Council (researcher-administered). Participants were graded from 1 to 5, as follows:

Grade 1: not troubled by breathlessness except with strenuous exercise

Grade 2: short of breath when hurrying on the level or walking up a slight hill

Grade 3: walks slower than most people on the level, stops after a mile or so, or stops after 15 min walking at own pace

Grade 4: stops for breath after walking around 100 yards or after a few minutes on level ground

Grade 5: too breathless to leave the house, or breathless when undressing

This has been well-validated against other measures of breathlessness and health status in diverse cardiorespiratory diseases and has an inter-observer agreement rate of 98\% (Mahler and Wells, 1988; Bestall et al., 1999).

Consistent with previous studies, the presence of moderate-to-severe breathlessness was defined as a MRC dyspnoea grade of $\geq 3$ (Bestall et al., 1999; Ahmed et al., 2012). Thus, the patient group was categorised into two clinically distinct subgroups according to MRC dyspnoea grade: 1) MRC dyspnoea grade $\leq 2$ (mild or no breathlessness) and 2) MRC dyspnoea grade $\geq 3$ (moderate-to-severe breathlessness).

\subsubsection{Perceived breathlessness intensity over the past 24 hours}

Perceived breathlessness intensity was measured by asking patients to record how much shortness of breath they had experienced 'over the past 24 hours' on a numerical rating scale (NRS) of 0 (none) to 10 (worst imaginable). The NRS has been shown to be a valid method of measuring present and usual breathlessness in patients with chronic respiratory disease (Gift and Narsavage, 1998). 


\subsubsection{Mastery domain of the Chronic Respiratory Questionnaire (CRQ)}

Patients' perceived ability to cope with or 'master' their breathlessness with respect to the 'last 2 weeks' was measured using the 'mastery' domain of the chronic respiratory questionnaire selfadministered scale (CRQ-SAS) (Guyatt et al., 1987). This domain consists of 4 items, rated on a 7point Likert scale from 1 (low mastery) to 7 (high mastery), with overall mastery calculated as the mean of the 4 items. It has been shown to be a valid and reliable measure of mastery in COPD patients (Cronbach's alpha $=0.83$ to 0.88 ) (Wijkstra et al., 1994). Patients only completed this questionnaire if they scored 1 or more on the breathlessness NRS.

\subsubsection{Sleep and psychological measures}

\subsubsection{Pittsburgh Insomnia Rating Scale-2 (PIRS-2)}

Subjective sleep quality was measured using the 2-item version of the 'Pittsburgh Insomnia Rating Scale (PIRS-2)', derived from the original 65-item version (Moul et al., 2002). Each item is rated on a 4-point Likert scale and total scores range from 0 (good) to 6 (poor).

\subsubsection{Perceived Stress Scale -10}

Perceived stress was measured using the 'Perceived Stress Scale-10 (PSS-10)'. This is a 10-item measure of the degree to which situations in one's life are perceived as stressful, with reference to 'the last month'. Each item is rated on a Likert-type scale and total scores range from 0 (low) to 40 (high). Validity and internal reliability (Cronbach's alpha $=0.78$ ) have been demonstrated in a US population study (Cohen and Williamson, 1988).

\subsubsection{Hospital Anxiety and Depression Scale (HADS)}

The 'Hospital Anxiety and Depression Scale (HADs)' was used to screen for anxiety and depression with reference to 'the past week'. This is a 14-item measure of psychological distress, with separate subscales for anxiety (HADs-A: 7-items, 0-21) and depression (HADS-D: 7-items, 0-21). It has been shown to be a valid and reliable measure in patient populations (Zigmond and Snaith, 1983). 


\subsubsection{Short Warwick-Edinburgh Mental Wellbeing Scale (SWEMWBS)}

Wellbeing was measured using the short-form of the Warwick-Edinburgh Mental Wellbeing Scale (SWEMWBS), a 7-item measure of positive mental wellbeing with reference to 'the last 2 weeks'. Each item is rated from 1 to 5 on a Likert-type scale. The total metric score ranges from 7 (low wellbeing) to 35 (high wellbeing). Validity and reliability have been demonstrated in a Scottish population study (Stewart-Brown et al., 2009).

\subsubsection{Measurement of compliance with the salivary cortisol sampling protocol} Compliance with the protocol for saliva collection was assessed by analysing participants' selfreported saliva collection times relative to their self-reported waking time, and assessing whether it was consistent with the protocol instructions. In addition, wrist actimeters (Actiwatch 2, Philips Respironics, UK) were used to check the accuracy of the self-reported waking time in 38 out of the 62 patients and in 42 out of the 50 healthy controls. These participants were instructed to apply the devices to their wrists the night before saliva collection. Actigraphy data were downloaded and analysed using Respironics Actiware version 6.0.4. In line with recommendations from Boyne et al., 2013, actigraph awakening times were scored by the human eye rather than the computer algorithm. The lead researcher scored awakening times and another author verified at least $10 \%$ of these.

Participants were considered to have deviated from the protocol if: 1) one or more of their samples were collected $>1$ hour outside the time specified by the protocol (Cohen et al., 2006), or 2 ) they did not record the collection time for one or more samples, or 3) they had one or more missing samples. 


\section{Statistical analysis}

\subsection{Mean diurnal cortisol slope with respect to health status}

To investigate our primary hypothesis that the DCS for patients with moderate-to-severe breathlessness ( $M R C \geq 3$ ) was different from that for patients with mild/no breathlessness and from that of healthy controls, we used a two-stage analysis. First, we calculated the DCS for each patient using linear regression of log-transformed cortisol levels against collection time across the 2 days (6 samples). Second, we assessed whether there was a difference in mean DCS between the three study groups (MRC $\leq 2$ versus $M R C \geq 3$ versus healthy) using ANCOVA, adjusting for age, gender and socioeconomic status. Pairwise comparisons were subsequently conducted using unpaired t-tests, adjusting for multiple comparisons using the Bonferroni test. ANCOVA model assumptions were checked by investigating the distribution of residuals graphically, using histograms and scatterplots.

For the patient group, in order to explore potential mediators and confounders of the relationship between DCS and health status, DCS was further explored in relation to other clinical and psychological variables relevant to breathlessness and/or cortisol, using unpaired t-tests for categorical variables and correlation for ordinal and continuous variables (Pearson if normally distributed and Spearman if non-normally distributed or ordinal).

All cortisol values were log-transformed to achieve approximately normal distributions. For descriptive purposes, raw cortisol data (nmol/L) are used for presentation within tables, summarised using both means and standard deviations (SD) and medians and interquartile ranges (IQRs).

\subsection{Diurnal slope categories with respect to health status}

In order to describe the different diurnal cortisol cycle patterns contributing to the mean diurnal cortisol slope, individual diurnal cortisol slopes were categorised, using the methodology employed by Smyth et al., (1997), as follows: 1) consistent declining, 2) consistent flat, 3) consistent ascending or 4) inconsistent. This analysis was limited to the 'MRC dyspnoea grade $\geq 3$ ' and 'healthy control' 
groups due to inadequate numbers in the 'MRC dyspnoea grade $\leq 2$ ' group. The difference in diurnal cycle category frequency between the two groups was assessed using a Fisher's Exact Test.

The consistency of the slope over the two days was analysed for each individual by comparing the slopes for day 1 and day 2. If the absolute difference between the slopes for day 1 and day 2 was greater than 0.08 (equivalent to 1 SD from the mean of the day-difference for the group as a whole), cycles were categorised as 'inconsistent' (Smyth et al., 1997). The value of 0.08 was obtained for the SD of the mean day-difference in both the 'MRC dyspnoea $\geq 3$ ' and 'healthy control' groups, allowing it to be used as the 'cut-off' value for both groups. Slopes were also categorised as 'inconsistent' if the direction of their slopes differed across the two days.

For individuals categorised as having consistent slopes across the 2 days, an aggregated slope value for the 2 days was calculated by regressing the 6 cortisol values over the 2 days on sample collection time (Sephton et al., 2000). Based on this value, the consistent slopes were sub-categorised as 'declining', 'flat' or 'ascending', represented by slopes $\leq-0.05,>-0.05$, or $>0$, respectively (Smyth et al., 1997).

\subsection{Handling of salivary cortisol protocol deviations}

The DCS was calculated in participants with minor protocol deviations if accommodation could be made for these deviations in the slope calculation: 1) in the case of mistimed samples, the actual saliva collection times rather than the protocol-stipulated collection times were used in the slope calculation, 2) in the case of missing samples, the slope was calculated using the available saliva samples, recognizing that a minimum of 2 data points is necessary for slope calculation, 3 ) where sample times were unrecorded, the protocol-stipulated time, as printed on the pre-labelled collection tubes, was used. The impact of minor protocol deviations on mean DCS was analysed using an unpaired t-test, comparing the mean DCS of fully-compliant participants with that of protocol-deviant participants. 


\section{Results}

\subsection{Compliance with the saliva collection protocol}

In the patient group, of the 366 commissioned saliva samples from 61 patients, 330 (90.2\%) were collected within 1 hour of the time stipulated by the protocol, $3(0.8 \%)$ were missing and $15(4.1 \%)$ had no collection time recorded. In regard to the self-reported awakening time, which was the anchor for the subsequent collection times, this was within 15 minutes of the objective actigraphyderived awakening time on $78 \%$ of the days recorded $(n=38)$.

Protocol-compliance in the patient group is summarized at a participant-level in Figure 1. Forty-four participants $(44 / 61,72.1 \%)$ were compliant for all samples within one hour of the stipulated protocol times. Seventeen participants $(17 / 61,26.2 \%)$ had some form of protocol deviation. One of these was excluded from data analysis from the outset due to major protocol deviation. The other 16 participants had evidence of only minor protocol deviation.

All 16 of the participants with evidence of minor protocol deviation were included in the mean DCS analysis, with accommodation made for missing or mistimed samples in the aggregated 2-day slope calculation. There was no significant difference in mean DCS between the fully compliant participants ( $n=44$; mean DCS $=-0.065)$ and the participants with minor protocol deviations $(n=16$; mean DCS $=-0.059 ; t=0.391, p=0.70$ ), validating the decision to include participants with minor protocol deviations in further analyses.

Compliance for the healthy control group is reported elsewhere (Evans et al. 2007). Due to high levels of compliance in this group, all healthy participants were included in the final analysis.

\subsection{Demographic, clinical and psychometric characteristics of the samples}

The demographic, clinical and psychometric characteristics for the three study groups are presented in Table 1. 
Across the three groups, there were differences in age, gender and subjective socio-economic status. Proportion of females and subjective socioeconomic status were higher in the healthy control group, relative to the patient groups. Mean age was lower in the 'MRC dyspnoea grade $\leq 2$ ' group relative to the other groups.

As expected, MRC dyspnoea grade subgroups differed with respect to several clinical and psychometric variables. Karnofsky performance status (KPS) and mastery over breathlessness (CRQ domain) were higher in those with a MRC grade $\leq 2$, whilst perceived breathlessness intensity (NRS), anxiety (HADs-A) and depression (HADs-D) levels were higher in those with a MRC grade $\geq 3$. Furthermore, patients with a MRC grade $\leq 2$ had predominantly malignant disease ( $81.8 \%)$, whereas patients with a MRC grade $\geq 3$ had predominantly non-malignant disease (71.4\%). Self-reported saliva collection protocol compliance rate was similar across MRC dyspnoea grade subgroups. 
Table 1. Demographic, clinical and psychometric characteristics of the three study groups

\begin{tabular}{|c|c|c|c|}
\hline & $\begin{array}{l}\text { MRC dyspnoea } \\
\text { grade } \geq 3 \\
\text { ( } n=49)\end{array}$ & $\begin{array}{l}\text { MRC dyspnoea } \\
\text { grade } \leq 2 \\
(n=11)\end{array}$ & $\begin{array}{l}\text { Healthy } \\
\text { control } \\
(n=50)\end{array}$ \\
\hline & $\mathrm{N}(\%)$ & $\mathrm{N}(\%)$ & $\mathrm{N}(\%)$ \\
\hline Gender (male) & $29(59.2)$ & $4(36.4)$ & $16(32.0)$ \\
\hline Site (hospital) & $38(77.6)$ & $2(18.2)$ & - \\
\hline Malignant disease & $14(28.6)$ & $9(81.8)$ & - \\
\hline \multicolumn{4}{|l|}{ Main diagnosis: } \\
\hline COPD & $11(22.4)$ & 0 & \\
\hline COPD and other cardiopulmonary disease & $8(16.3)$ & 0 & \\
\hline Primary lung cancer alone & $8(16.3)$ & $1(9.1)$ & \\
\hline Chronic heart failure alone & $7(14.3)$ & 0 & \\
\hline Pulmonary fibrosis $\left(1^{\circ}\right.$ or $\left.2^{\circ}\right)$ & $5(10.2)$ & 0 & \\
\hline Metastatic cancer (non-lung primary) & $5(10.2)$ & $6(54.5)$ & \\
\hline Neuromuscular disease & $3(6.1)$ & $2(18.2)$ & \\
\hline Haematological malignancy & 0 & $2(18.2)$ & \\
\hline Other pulmonary diseases & $2(4.1)$ & 0 & \\
\hline \multirow[t]{4}{*}{ MRC Dyspnoea grade (G1-5): } & & & - \\
\hline & G3: 10 (20.4) & G1: 4 (34.4) & \\
\hline & G4: 19 (38.8) & G2: 7 (63.6) & \\
\hline & G5: 20 (40.8) & & \\
\hline \multicolumn{4}{|l|}{ Charlson Co-Morbidity Index } \\
\hline $0-1$ & $18(36.7)$ & $1(9.1)$ & \\
\hline $2-3$ & $14(28.6)$ & $4(36.4)$ & \\
\hline $4-5$ & $7(14.3)$ & 0 & \\
\hline 6-7 & $6(12.2)$ & $5(45.5)$ & \\
\hline$>8$ & $4(8.2)$ & $1(9.1)$ & \\
\hline \multicolumn{3}{|l|}{ Smoking History: } & - \\
\hline Never Smoker & $6(12.2)$ & $6(54.5)$ & \\
\hline Ex-smoker & $41(83.7)$ & $5(45.5)$ & \\
\hline Current smoker & $2(4.1)$ & 0 & \\
\hline \multicolumn{3}{|l|}{ Oxygen therapy: } & - \\
\hline No & $35(71.4)$ & $11(100)$ & \\
\hline SBOT & $8(16.3)$ & 0 & \\
\hline LTOT & $6(12.2)$ & 0 & \\
\hline \multicolumn{3}{|l|}{ Relevant medications: } & - \\
\hline None & $11(22.4)$ & $4(36.4)$ & \\
\hline Steroid-based inhaler only & $14(28.6)$ & $1(9)$ & \\
\hline Othert & $11(22.4)$ & $5(45.5)$ & \\
\hline Steroid-based inhaler and other & $11(22.4)$ & $1(9)$ & \\
\hline \multicolumn{3}{|l|}{ Relevant medical information: } & - \\
\hline Type I or II diabetes mellitus (yes) & $8(16.3)$ & $2(18.2)$ & \\
\hline Past/present psychiatric diagnosis (yes) & $5(10.2)$ & $1(9.1)$ & \\
\hline Systemic corticosteroids past year (yes) & $23(46.9)$ & $4(36.4)$ & \\
\hline \multirow[t]{2}{*}{ Protocol compliance (yes) ${ }^{\ddagger}$} & $35(71.4)$ & $9(81.8)$ & - \\
\hline & Mean (SD) & Mean (SD) & Mean (SD) \\
\hline Age & $74.92(8.26)$ & $65.00(9.14)$ & $73.98(6.96)$ \\
\hline Subjective SES ladder (1-10) & $5.71(1.74)^{*}$ & $6.36(1.43)$ & $7.00(1.76)$ \\
\hline KPS $(10-100)$ & $67.14(9.57)$ & $80.00(7.75)$ & - \\
\hline Breathlessness NRS (0-10) & $5.84(2.36)$ & $1.18(1.40)$ & - \\
\hline CRQ-Mastery (1-7) & $4.35(1.29)$ & $6.04(0.68)^{* *}$ & - \\
\hline PSS-10 (0-40) & $18.37(6.84)$ & $13.64(8.98)$ & - \\
\hline HADS-A (0-21) & $7.8(4.30)$ & $4.64(4.88)$ & - \\
\hline HADS-D (0-21) & $7.73(3.33)$ & $4.55(2.54)$ & - \\
\hline SWEMWBS (7-35) & $22.46(4.10)^{*}$ & $23.99(3.34)$ & - \\
\hline
\end{tabular}


PIRS-2 (0-6) 3.00 (1.86)

2.36 (1.69)

Abbreviations: MRC, Medical Research Council; SBOT, short-burst oxygen therapy; LTOT, long-term oxygen therapy; CBIS, Cambridge Breathlessness Intervention Service; SES, socioeconomic status; KPS, Karnofsky Performance Status; NRS, numerical rating scale; CRQ, Chronic Respiratory Questionnaire; PSS-10, Perceived Stress Scale-10 item version; HADS-A, Hospital Anxiety and Depression Scale- Anxiety domain; HADS-D, Hospital Anxiety and Depression Scale- Depression domain; SWEMWBS, Short Warwick-Edinburgh Mental Wellbeing Scale; Pittsburgh Insomnia Rating Scale 2-item version. Symbols: †Other relevant medications: antidepressants, hormone therapy, opioids, immunosuppressant therapy, chemo-radiotherapy, anti-psychotics and insulin; ¥all samples taken within one-hour of the protocol time (self-reported); ${ }^{*} n=48$, missing data from 1 participant; ${ }^{* *} n=6$, only completed by those with a score $>0$ for breathlessness NRS 


\subsection{Mean diurnal cortisol slope with respect to health status}

Salivary cortisol values ranged from 0.10 to $47.31 \mathrm{nmol} / \mathrm{l}$ across the three groups. Log-transformed cortisol profiles for the three study groups are summarised in Figures 2 and 3. Mean and median raw cortisol values for each time point, as well as mean and median DCS, are presented within Figure 2.

A one-way between-subjects ANCOVA, adjusting for age, gender and socioeconomic status, demonstrated a significant main effect of study group ( 3 levels: $M R C \leq 2, M R C \geq 3$, and healthy control) on $\operatorname{DCS}\left[F(2,103)=45.64, p<0.001, \eta^{2}=0.47\right]$, with this model explaining $51.1 \%$ of the variability in DCS (adjusted $\mathrm{R}^{2}=0.511$ ) and study group explaining $47 \%$ of the variability in DCS (See Table 2 ).

Table 2 One-way between-subjects ANCOVA for diurnal cortisol slope by study group (MRC $\geq 3$ Vs MRC $\leq 2$ Vs healthy), with age, gender and socio-economic status as covariates ${ }^{*}$

\begin{tabular}{|c|c|c|c|c|c|}
\hline Source & df & MS & $\mathbf{F}$ & p-value & B coefficient $(95 \% \mathrm{Cl})$ \\
\hline \multirow[t]{3}{*}{ Study group } & 2 & 0.117 & 45.636 & 0.000 & $M R C \geq 3: 0.107(0.085,0.129)$ \\
\hline & & & & & $\mathrm{MRC} \leq 2: 0.048(0.012,0.084)$ \\
\hline & & & & & Healthy control: 0 \\
\hline Gender & 1 & $3.232 E-5$ & 0.013 & 0.911 & $0.001(-0.02,0.22)$ \\
\hline Age & 1 & 0.001 & 0.510 & 0.477 & $0.000(-0.001,0.002)$ \\
\hline Socio-economic status & 1 & 0.002 & 0.674 & 0.413 & $-0.002(-0.008,0.003)$ \\
\hline Intercept & 1 & 0.014 & 5.494 & 0.002 & $-0.183(-0.299,-0.066)$ \\
\hline Residual & 103 & 0.003 & & & \\
\hline
\end{tabular}

*Note: $\mathrm{R}^{2}=0.534$ (adjusted $\mathrm{R}^{2}=0.511$ ), $\mathrm{p}<0.001$; adjustments based on the following covariate values: age $=73.5$ and socio-economic status=6.367; Abbreviations: df, degrees of freedom; MS, Mean Squares; $\mathrm{Cl}$, Confidence Interval

Post-hoc pair-wise comparisons, adjusting for multiple comparisons using the Bonferroni test, showed that there was a significant difference in mean DCS between each of the three groups. The mean DCS for the MRC $\geq 3$ group was significantly flatter than that of the MRC $\leq 2$ group and that of the healthy control group ( $p<0.001$ for both comparisons). Furthermore, the DCS in the MRC $\leq 2$ group was significantly flatter than that of the healthy control group $(p=0.024)$.

Further subgroup analysis for the patient group indicated that there was no significant difference in mean DCS between subgroups when categorised according to steroid inhaler use, systemic 
corticosteroid history, disease category (malignant versus non-malignant) and study site (see Table

3a). Furthermore, apart from MRC dyspnoea grade, DCS did not correlate significantly with any of

the clinical and psychological variables known to be related to breathlessness and/or stress (see

Table 3b).

The results of additional statistical analyses, not described here, may be found in the Appendix.

Table 3: Relationship of the diurnal cortisol slope (DCS) with other clinical and psychological variables in the patient group, with a) showing the mean DCS in relation to different subgroup characteristics and b) showing the correlation of DCS with continuous and ordinal variables

a) Mean DCS (SD) by subgroup characteristic*

\begin{tabular}{llll}
\hline & Yes & No & Statistical difference \\
\hline Steroid inhaler & $-0.058(0.043)$ & $-0.067(0.060)$ & $\mathrm{t}=0.629, \mathrm{p}=0.53$ \\
& $\mathrm{~N}=27$ & $\mathrm{~N}=33$ & $\mathrm{t}=0.091, \mathrm{p}=0.93$ \\
\hline Systemic corticosteroids in the & $-0.062(0.045)$ & $-0.064(0.059)$ & \\
past year & $\mathrm{N}=27$ & $\mathrm{~N}=33$ & $\mathrm{t}=1.46, \mathrm{p}=0.15$ \\
\hline Malignant disease & $-0.075(0.060)$ & $-0.055(0.047)$ & $\mathrm{t}=1.46, \mathrm{p}=0.16^{\dagger}$ \\
\hline Hospital site & $\mathrm{N}=23$ & $\mathrm{~N}=37$ & \\
\end{tabular}

*Two-tailed unpaired t-tests conducted to compare means between subgroups, with equal variances assumed unless otherwise stated; †equal variances not assumed (Welch's t-test)

b) Correlation of the DCS (and MRC dyspnoea grade) with other variablest

\begin{tabular}{llllllllllll}
\hline & & $\begin{array}{l}\text { MRC } \\
\text { dyspnoea } \\
\text { grade }\end{array}$ & $\begin{array}{l}\text { Breath- } \\
\text { lessness } \\
\text { NRS }\end{array}$ & $\begin{array}{l}\text { CRQ- } \\
\text { Mastery }\end{array}$ & $\begin{array}{l}\text { PSS- } \\
\mathbf{1 0}\end{array}$ & $\begin{array}{l}\text { HADS } \\
\text {-A }\end{array}$ & $\begin{array}{l}\text { HADS } \\
\text {-D }\end{array}$ & $\begin{array}{l}\text { PIRS- } \\
\mathbf{2}\end{array}$ & $\begin{array}{l}\text { SWEM } \\
\text {-WBS }\end{array}$ & KPS & CCI \\
\hline DCS & $r$ & $0.29(\mathrm{rs})$ & 0.23 & -0.15 & 0.072 & 0.087 & 0.18 & 0.20 & 0.074 & -0.23 & $-0.002(\mathrm{rs})$ \\
& $\mathrm{p}$ & 0.027 & 0.074 & 0.27 & 0.58 & 0.51 & 0.17 & 0.14 & 0.58 & 0.082 & 0.99 \\
& $\mathrm{~N}$ & 60 & 60 & 55 & 60 & 60 & 60 & 59 & 59 & 60 & 60 \\
\hline MRC & $\mathrm{rS}$ & 1 & 0.59 & -0.45 & 0.36 & 0.29 & 0.35 & -0.03 & -0.29 & 0.75 & -0.24 \\
Dyspnoea & $\mathrm{p}$ & - & $<0.001$ & $<0.001$ & 0.005 & 0.026 & 0.006 & 0.84 & 0.029 & $<0.001$ & 0.060 \\
grade & $\mathrm{N}$ & 60 & 60 & 55 & 60 & 60 & 60 & 60 & 59 & 60 & 60 \\
\hline
\end{tabular}

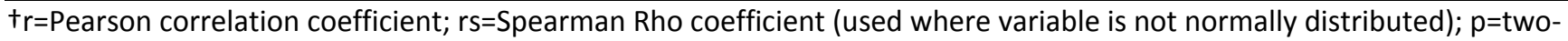
tailed significance test; $\mathrm{N}=$ sample number. Abbreviations: DCS, diurnal cortisol slope; MRC, Medical Research Council; NRS, numerical rating scale; $C R Q$, Chronic Respiratory Questionnaire; PSS-10, Perceived Stress Scale-10; HADS-A, Hospital Anxiety and Depression Score-Anxiety subscale; HADS=D, Hospital Anxiety and Depression Score-Depression subscale; PIRS-2, Pittsburgh Insomnia Rating Scale-2; SWEMWBS, Short Warwick-Edinburgh Mental Wellbeing Scale; KPS, Karnofsky Performance Status; $\mathrm{CCl}$, Charlson Comorbidity Index 


\subsection{Description of diurnal cortisol slope categories with respect to health status}

The DCS category analysis was confined to patients with a MRC dyspnoea grade $\geq 3(n=47)$. Two patients were excluded as it was not possible to calculate their slope on each of days 1 and 2, a prerequisite for this analysis.

There was a significant difference between patient (MRC dyspnoea $\geq 3$ ) and healthy groups in relation to the frequency of the four diurnal cycle categories (Fisher's exact test=16.337, $p<0.001$ ), with a higher frequency of aberrant profiles (flat, ascending or inconsistent) in the 'MRC dyspnoea $\geq 3^{\prime}$ group (See Figure 3). In the patient group, the proportions of descending, flat, ascending and inconsistent profiles were $48.9 \%, 19.1 \%, 4.3 \%$ and $27.7 \%$ respectively. By contrast, in the healthy group, $80 \%$ had descending profiles, no participant had a flat or ascending profile and $20 \%$ had inconsistent profiles.

\section{Discussion}

We have described the salivary diurnal cortisol profile, a surrogate marker of HPA axis activity, in patients suffering from moderate-to-severe breathlessness (MRC $\geq 3$ ) secondary to a range of cardiorespiratory diseases. We have shown that patients with moderate-to-severe breathlessness had significantly flatter diurnal cortisol slopes compared to healthy adults. We have also shown that patients with moderate-to-severe breathlessness had a mixture of declining, flat and ascending slopes, a pattern significantly different to that seen in the healthy adult group. Furthermore, we have shown that patients with moderate-to-severe breathlessness had significantly flatter slopes compared to patients with mild/no breathlessness (MRC $\leq 2$ ). Of the many clinical and psychological variables measured, MRC dyspnoea grade was the only variable significantly associated with DCS, with a higher MRC dyspnoea grade being associated with a flatter DCS. 


\subsection{Relationship between DCS and disease}

Our study has shown that both patient subgroups (breathless and non-breathless) had significantly flatter slopes compared to the healthy control group, after controlling for differences in age, gender and socio-economic status. The finding of flatter diurnal slopes in a patient population is consistent with the literature. For example, flatter slopes have previously been reported in specific disease groups, including metastatic breast cancer (Abercrombie et al., 2004) and diabetes mellitus (Hackett et al., 2014), relative to healthy controls. To the authors' knowledge, this is the first time that flatter slopes have been identified in a mixed patient population, where the patient groups were defined according to symptoms rather than underlying disease.

The mean DCS was markedly flatter in patients with moderate-to-severe breathlessness (MRC $\geq 3$ ) in comparison with the healthy control group. Given that the mean DCS for the healthy control group is comparable with slope magnitudes reported for other healthy populations in the literature (Stone et al., 2001; Ice et al., 2004; Kumari et al., 2011 ), it is reasonable to conclude that the substantially flatter mean slope in the breathless patient group points to HPA axis dysregulation. In our study, this flatter mean DCS appears to be due to the combined effect of lower 3-hour and higher 12-hour cortisol values within patients. It is also consistent with the higher prevalence of flat/ascending profiles in this sample relative to the healthy control group.

In the patients with moderate-to-severe breathlessness, the proportions of descending, flat, ascending and inconsistent profiles were $48.9 \%, 19.1 \%, 4.3 \%$ and $27.7 \%$ respectively. To date, the different types of cortisol profile patterns that exist within patient populations have not been welldescribed. Furthermore, meaningful comparison between the few available studies is virtually impossible in the absence of agreed classification and measurement criteria. Sephton et al. (2000) categorised 3-day cortisol slopes in patients with metastatic breast cancer (mean age: 52.3 years) into three categories, by visual inspection of individual plotted cortisol profiles. They found that $37 \%$ of patients had declining profiles with an early peak at $08.00 \mathrm{am}, 49 \%$ had late peaks in cortisol at 
noon or afternoon, and $14 \%$ had flat profiles. Though difficult to compare, it is noteworthy that the proportion of flat slopes is similar to that in our breathless patient sample. Woods et al. (2008) also visually inspected individual cortisol plots in order to categorise 1-day diurnal cycles in patients with dementia ( 2 cohorts, mean age: 81 years). In one cohort ( $n=21), 52.4 \%$ had declining cycles, $14.3 \%$ had flat cycles, and $33.3 \%$ had a rise in the evening cortisol level. In the other cohort $(n=16), 43.7 \%$ had declining cycles, $14.5 \%$ had flat cycles, and $35.5 \%$ had a rise in the evening cortisol level. These are similar to our findings in that only approximately half of the patients had declining cycles.

\subsection{Relationship between DCS and breathlessness}

Our study has also shown that patients with moderate-to-severe breathlessness (MRC $\geq 3$ ) had significantly flatter diurnal cortisol slopes than patients with mild or no breathlessness (MRC $\leq 2$ ), after controlling for age, gender and socioeconomic status. Furthermore, correlational analysis indicated that MRC dyspnoea grade was the only clinical variable significantly associated with DCS. As MRC dyspnoea grade measures 'functional disability due to breathlessness', these findings suggest that patients with greater functional disability due to breathlessness have flatter diurnal cortisol slopes. It is noteworthy that DCS did not correlate significantly with measures of perceived breathlessness intensity ('breathlessness NRS) or level of mastery over breathlessness (CRQ), though there was a trend towards an association with perceived breathlessness intensity. This may suggest that DCS is more related to the functional impact of breathlessness rather than its perceptual or psychological dimensions.

Whilst MRC dyspnoea grade measures the functional disability imposed by breathlessness (Bestall et al., 1999), the literature suggests that it represents far more than this. For example, it has been shown to be related to both disease-specific and generic health-related quality of life in COPD patients (Hajiro et al., 1999), and has been shown to be a significant predictor of survival in both COPD (Nishimura et al., 2002) and idiopathic pulmonary fibrosis (Nishiyama et al., 2010), independent of lung function. It was also shown to be a significant predictor of 2-year and 10-year 
mortality in a population study of elderly community-dwelling adults, independent of age, gender and underlying cardiorespiratory disease (Ahmed et al., 2012). Thus, the association between DCS and MRC dyspnoea category could reflect differences in functional impairment, health-related quality of life or prognosis between patients with MRC dyspnoea grades $\leq 2$ and grades $\geq 3$.

In support of our findings, diurnal cortisol rhythm has already been linked to constructs related to MRC dyspnoea grade in other populations. The association between flatter diurnal cortisol slopes and higher MRC dyspnoea grade is consistent with the growing literature demonstrating flatter diurnal slopes in association with shorter survival in cancer (Sephton et al., 2000; Cohen et al., 2012; Sephton et al., 2013). It is also consistent with the strong evidence in the literature for a relationship between DCS and functional impairment in older adults. In a meta-analysis of 6 adult cohorts (50-92 years; $n=>2000$ ), a larger diurnal drop (difference between morning and evening cortisol) was found to be associated with faster walking speed and quicker chair rise, suggesting that smaller diurnal drops (or flatter diurnal slopes) are associated with worse physical performance (Gardner et al., 2013). In keeping with these findings, blunted diurnal variation in cortisol was also shown to be associated with greater frailty in a cohort of 200 elderly participants aged 80-90 years (Varadhan et al., 2008).

\subsection{Potential mechanisms}

Given the cross-sectional nature of the study, the direction of the relationship between MRC dyspnoea grade and diurnal slope cannot be ascertained, and causality cannot be proven. One possibility is that HPA axis dysregulation and functional disability due to breathlessness are caused by the same underlying disease process. A second possibility is that functional impairment due to breathlessness contributes to HPA axis dysregulation. Alternatively, a third possibility is that HPA axis dysregulation, whatever its cause, contributes to functional disability due to breathlessness.

Chronic systemic inflammation is one process which might explain the relationship between MRC dyspnoea grade and diurnal slope, in each of the postulated directions. This process has been 
identified in many chronic diseases commonly associated with breathlessness, such as COPD (Gan et al., 2004) and cancer (Laird et al., 2013). In these contexts, it may contribute to disability due to breathlessness (MRC dyspnoea grade) by inducing skeletal muscle atrophy (Cohen et al., 2015). It may also contribute to HPA axis dysregulation through excessive stimulation of the HPA axis by cytokines, with consequent glucocorticoid resistance (Rosenblat et al., 2014). Furthermore, disability due to breathlessness or a high MRC dyspnoea grade may itself promote chronic inflammation, a hypothesis supported by the inverse relationship between C-reactive protein and physical activity in several population cohort studies (Kasapis and Thompson, 2005), as well as the anti-inflammatory effects of exercise in diverse health populations (Nicklas and Brinkley, 2009). Resultant HPA axis dysregulation may in turn promote and perpetuate chronic systemic inflammation due to loss of effective cortisol-mediated anti-inflammatory effects (Silverman and Sternberg, 2012).

The negative findings in this study offer valuable insight into potentially relevant mechanisms underlying HPA axis dysregulation in this patient group. Counter to our expectations, the DCS was not found to be related to any of the psychological variables measured, including depression and perceived stress. This is consistent with the findings of other studies of patient populations (Sephton et al., 2000; Weinrib et al., 2011; Sephton et al., 2013) and at odds with findings in healthy populations where a flatter diurnal slope has been linked with psychosocial stress or chronic stress conditions (Ehlert et al., 2001; Adam et al., 2006). This might suggest that the effect of physical disease on the HPA axis overrides the effects of psychological factors in the context of advanced disease. Furthermore, there was no significant difference in DCS between malignant and nonmalignant subgroups, suggesting that HPA axis dysregulation is a non-specific phenomenon, common to a range of disease types, with more research warranted in non-malignant populations. Importantly, comparison of DCS between steroid inhaler users and non-users indicated no significant difference, suggesting that steroid inhalers did not have a large effect on the diurnal slope in this patient population. 


\subsection{Strengths and limitations}

A strength of this study is the analysis and presentation of the DCS both as a continuous variable (summarised by the group mean) and as a categorical variable (summarised by slope steepness and direction). The mean diurnal slope can hide significant inter-individual variation within a sample. Furthermore, a flatter mean diurnal slope may arise for a number of reasons (e.g. a combination of descending and ascending slopes), such that it is difficult to interpret its meaning when presented in isolation. Using our dual approach, we were able to confidently demonstrate that the flatter mean DCS in patients with moderate-to-severe breathlessness was due to a significantly higher proportion of flat, ascending and inconsistent slopes in this group compared to the healthy control group. A further strength is the consistency of the relationship between MRC dyspnoea grade and DCS, regardless of the statistical approach used. A final strength is its novelty in measuring diurnal cortisol patterns in patients receiving palliative and supportive care services, a patient group with a significant burden of both psychological and physical symptoms; this has allowed further insight into the complex mind-body relationship.

Several limitations must be taken into consideration when interpreting these findings. First, compliance with the saliva collection protocol was measured by self-report, such that reported compliance may not accurately reflect actual compliance. The wrist actimetry data would suggest that self-reporting was reasonably accurate in the majority of cases, however. Secondly, though there was no significant difference in mean DCS between the fully compliant participants and the participants with minor protocol deviations, the sample size for this subgroup analysis was small; thus, we cannot be certain of the impact of minor protocol deviations on the overall results.

A second limitation was the use of a historical healthy control group, which was recruited through a separate study, conducted in a different geographical area; this may have introduced selection bias. Despite this, it is important to note that both groups represented community-dwelling older adults and that samples from both groups were analysed in the same laboratory using the same assay. 
Whilst differences in socio-economic status and gender were controlled for in the analysis, potential differences in unmeasured psychological variables were not. It is important to bear in mind that the DCS appeared to be unrelated to psychological status in the patient group, however, suggesting that psychological variables were not important covariates.

Due to the small sample size, the correlation and subgroup analyses must be interpreted with caution. In particular, the comparison between breathless (MRC $\geq 3$ ) and non-breathless (MRC $\leq 2$ ) patients was limited by the small sample size of the non-breathless subgroup (MRC $\leq 2 ; n=11$ ), which may not have been a sufficiently representative sample. In addition, in order to reduce patientburden, we used a 2-item measure of sleep quality, which is not yet supported by validation studies. The small sample size also limited our diurnal slope category analysis, for which we used arbitrary cut-off values to define slope categories; a larger sample size may have allowed modelling of diurnal profile patterns from the data using a mixed modelling approach. Finally, this analysis is subject to the limitations of all case-control analyses in its inability to control for unknown confounders and to prove causation.

\subsection{Future work}

There are many questions left to be answered about the relationship of HPA axis function to both chronic breathlessness and chronic progressive disease. This study focused on salivary diurnal cortisol as a surrogate marker of HPA axis function but it would be important in future studies to measure other markers of HPA axis function, such as adrenocorticotrophin hormone (ACTH), to assess whether dysfunction occurs at the pituitary or adrenal level. Furthermore, the temporal relationship between chronic breathlessness and HPA axis dysfunction needs to be established, as well as the impact of disease characteristics such as breathlessness duration, hypoxia and prognosis on HPA axis function. 


\section{Conclusion}

In summary, this cross-sectional study provides novel evidence of HPA axis dysregulation in patients with moderate-to-severe breathlessness, manifesting as flatter diurnal cortisol decline slopes. The degree of dysregulation appears to relate to functional disability due to breathlessness, rather than psychological distress. Future longitudinal studies are necessary to explore the temporal relationship between breathlessness-induced disability and HPA axis dysregulation, as well as its prognostic implications.

\section{Acknowledgements}

The authors would like to thank the following people for their support of the study: all patients who participated; past and present staff of the Cambridge Breathlessness Intervention Service and the Palliative Care Department of Cambridge University Hospitals NHS Foundation Trust, particularly those who helped with recruitment and administration (in particular, Dr. Rosemary Wade, Julie Burkin, Pauline Kleanthous, Catherine Moffat, Rhys Hurst, Malika Harboun, Natalie Brown and Dr. Sarah Grove); staff at Arthur Rank House Hospice, Cambridge (in particular, Dr. Lorraine Petersen who was the principal investigator at the site, and Jude Buggs who helped with recruitment); Matthew Barclay (Department of Public Health and Primary Care, University of Cambridge) for providing statistical advice; Prof Phil Evans and Catherine Fredhoi (Department of Psychology, University of Westminster) for providing access to the heathy-control dataset; Morag Farquhar (Department of Public Health and Primary Care, University of Cambridge) for providing general advice and support; National Institute for Health Research (NIHR) for funding the study in the form of a doctoral research fellowship held by Dr. Richella Ryan (DRF-2012-05-702).

This article presents independent research funded by the National Institute for Health Research (NIHR: DRF-2012-05-702). The views expressed are those of the authors and not necessarily those of the NHS, the NIHR or the Department of Health. 


\section{References}

Abercrombie, H.C., Giese-Davis, J., Sephton, S. et al., 2004. Flattened cortisol rhythms in metastatic breast cancer patients. Psychoneuroendocrinology 29, 1082-1092.

Adam, E.K., Hawkley, L.C., Kudielka, B.M., Cacioppo, J.T., 2006. Day-to-day dynamics of experience-cortisol associations in a population-based sample of older adults. Proceedings of the National Academy of Sciences of the United States of America 103, 17058-17063.

Adler, N.E., Epel, E.S., Castellazzo, G., Ickovics, J.R., 2000. Relationship of subjective and objective social status with psychological and physiological functioning: preliminary data in healthy white women. Health psychology 19, 586-592.

Ahmed, T., Steward, J.a., O'Mahony, M.S., 2012. Dyspnoea and mortality in older people in the community: a 10-year follow-up. Age and ageing 41, 545-549.

Bestall, J.C., Paul, E.a., Garrod, R., Garnham, R., Jones, P.W., Wedzicha, J.a., 1999. Usefulness of the Medical Research Council (MRC) dyspnoea scale as a measure of disability in patients with chronic obstructive pulmonary disease. Thorax 54, 581-586.

Booth, S., Silvester, S., Todd, C., 2003. Breathlessness in cancer and chronic obstructive pulmonary disease: Using a qualitative approach to describe the experience of patients and carers. Palliative \& Supportive Care 1, 337-344.

Bower, J.E., Ganz, P.A., Dickerson, S.S., Petersen, L., Aziz, N., Fahey, J.L., 2005. Diurnal cortisol rhythm and fatigue in breast cancer survivors. Psychoneuroendocrinology 30, 92-100.

Boyne, K., Sherry, D.D., Gallagher, P.R., Olsen, M., Brooks, L.J., 2013. Accuracy of computer algorithms and the human eye in scoring actigraphy. Sleep Breath 17, 411-417.

Charlson, M., Pompei, P., Ales, K., MacKenzie, R., 1987. A new method of classifying prognostic comorbidity in longitudinal studies: development and validation. Journal of Chronic Diseases 40, 373-383.

Cohen, L., Cole, S.W., Sood, A.K., Prinsloo, S., Kirschbaum, C., Arevalo, J.M.G., Jennings, N.B., Scott, S., Vence, L., Wei, Q., Kentor, D., Radvanyi, L., Tannir, N., Jonasch, E., Tamboli, P., Pisters, L., 2012. Depressive symptoms and cortisol rhythmicity predict survival in patients with renal cell carcinoma: Role of inflammatory signaling. PLoS ONE 7, e42324 .

Cohen, S., Nathan, J.A., Goldberg, A.L., 2015. Muscle wasting in disease: molecular mechanisms and promising therapies. Nat Rev Drug Discov 14, 58-74.

Cohen, S., Schwartz, J.E., Epel, E., Kirschbaum, C., Sidney, S., Seeman, T., 2006. Socioeconomic status, race, and diurnal cortisol decline in the Coronary Artery Risk Development in Young Adults (CARDIA) Study. Psychosom Med 68, 41-50.

Cohen, S., Williamson, G., 1988. Perceived Stress in a Probability Sample of the United States. , in: SPACAPAN, S., OSKAMP, S. (Eds.), The Social Psychology of Health. Sage, Newbury Park, CA.

Dantzer, R., Meagher, M.W., Cleeland, C.S., 2012. Translational approaches to treatment-induced symptoms in cancer patients. Nat Rev Clin Oncol 9, 414-426. 
Ehlert, U., Gaab, J., Heinrichs, M., 2001. Psychoneuroendocrinological contributions to the etiology of depression, posttraumatic stress disorder, and stress-related bodily disorders: the role of the hypothalamus-pituitary-adrenal axis. Biological psychology 57, 141-152.

Evans, P., Forte, D., Jacobs, C., Fredhoi, C., Aitchison, E., Hucklebridge, F., Clow, A., 2007. Cortisol secretory activity in older people in relation to positive and negative well-being.

Psychoneuroendocrinology 32, 922-930.

Fletcher, C.M., Elmes, P.C., Fairbairn, A.S., Wood, C.H., 1959. The significance of respiratory symptoms and the diagnosis of chronic bronchitis in a working population. Br Med J 2, 257-266.

Gan, W.Q., Man, S.F., Senthilselvan, A., Sin, D.D., 2004. Association between chronic obstructive pulmonary disease and systemic inflammation: a systematic review and a meta-analysis. Thorax 59, 574-580.

Gardner, M.P., Lightman, S., Sayer, A.A., Cooper, C., Cooper, R., Deeg, D., Ebrahim, S., Gallacher, J., Kivimaki, M., Kumari, M., Kuh, D., Martin, R.M., Peeters, G., Ben-Shlomo, Y., 2013. Dysregulation of the hypothalamic pituitary adrenal (HPA) axis and physical performance at older ages: An individual participant meta-analysis. Psychoneuroendocrinology 38, 40-49.

Gift, A.G., Narsavage, G., 1998. Validity of the numeric rating scale as a measure of dyspnea. American journal of critical care 7, 200-204.

Guyatt, G.H., Berman, L.B., Townsend, M., Pugsley, S., Chambers, L.W., 1987. A measure of quality of life for clinical trials in chronic lung disease. Thorax, 42:773-778.

Hackett, R.a., Steptoe, A., Kumari, M., 2014. Association of diurnal patterns in salivary cortisol with type 2 diabetes in the Whitehall II study. The Journal of clinical endocrinology and metabolism 99, 4625-4631.

Hajiro, T., Nishimura, K., Tsukino, M., Ikeda, a., Oga, T., Izumi, T., 1999. A comparison of the level of dyspnea vs disease severity in indicating the health-related quality of life of patients with COPD. Chest 116, 1632-1637.

Ice, G.H., Katz-Stein, A., Himes, J., Kane, R.L., 2004. Diurnal cycles of salivary cortisol in older adults. Psychoneuroendocrinology 29, 355-370.

Karnofsky, D., Burchenal, J., 1949. The clinical evaluation of chemotherapeutic agents in cancer, In: MacCleod, CM. (eds.) Evaluation of chemotherapeutic agents. Columbia University Press New York, pp. 191-205.

Kasapis, C., Thompson, P.D., 2005. The effects of physical activity on serum C-reactive protein and inflammatory markers: a systematic review. J Am Coll Cardiol 45, 1563-1569.

Kim, H.J., Barsevick, A.M., Fang, C.Y., Miaskowski, C., 2012. Common biological pathways underlying the psychoneurological symptom cluster in cancer patients. Cancer Nurs 35, E1-E20.

Kumari, M., Shipley, M., Stafford, M., Kivimaki, M., 2011 Association of diurnal patterns in salivary cortisol with all-cause and cardiovascular mortality: Findings from the Whitehall II study. Journal of Clinical Endocrinology and Metabolism 96, 1478-1485. 
Laird, B.J., McMillan, D.C., Fayers, P., Fearon, K., Kaasa, S., Fallon, M.T., Klepstad, P., 2013. The systemic inflammatory response and its relationship to pain and other symptoms in advanced cancer. Oncologist 18, 1050-1055.

Mahler, D.A., Wells, C.K., 1988. Evaluation of clinical methods for rating dyspnea. Chest 93, 580-586.

Miller, A.H., Ancoli-Israel, S., Bower, J.E., Capuron, L., Irwin, M.R., 2008. Neuroendocrine-immune mechanisms of behavioral comorbidities in patients with cancer. J Clin Oncol 26, 971-982.

Miller, G.E., Chen, E., Zhou, E.S., 2007. If it goes up, must it come down? Chronic stress and the hypothalamic-pituitary-adrenocortical axis in humans. Psychological bulletin 133, 25-45.

Moul, D., Pilkonis, P., Miewald, J., Carey, T., Buysse, D., 2002. Preliminary study of the test-retest reliability and concurrent validities of the Pittsburgh Insomnia Rating Scale (PIRS). Sleep 25, A246247.

Nicklas, B.J., Brinkley, T.E., 2009. Exercise training as a treatment for chronic inflammation in the elderly. Exerc Sport Sci Rev 37, 165-170.

Nishimura, K., Izumi, T., Tsukino, M., Oga, T., Nishimura, K., Izumi, T., Tsukino, M., Oga, T., 2002. Dyspnea is a better predictor of 5-year survival than airway obstruction in patients with COPD. Chest $121,1434-1440$.

Nishiyama, O., Taniguchi, H., Kondoh, Y., Kimura, T., Kato, K., Kataoka, K., Ogawa, T., Watanabe, F., Arizono, S., 2010. A simple assessment of dyspnoea as a prognostic indicator in idiopathic pulmonary fibrosis. European Respiratory Journal 36, 1067-1072.

Rosenblat, J.D., Cha, D.S., Mansur, R.B., McIntyre, R.S., 2014. Inflamed moods: a review of the interactions between inflammation and mood disorders. Prog Neuropsychopharmacol Biol Psychiatry 53, 23-34.

Sephton, S.E., Lush, E., Dedert, E.a., Floyd, A.R., Rebholz, W.N., Dhabhar, F.S., Spiegel, D., Salmon, P., 2013. Diurnal cortisol rhythm as a predictor of lung cancer survival. Brain, behavior, and immunity 30 Suppl, S163-170.

Sephton, S.E., Sapolsky, R.M., Kraemer, H.C., Spiegel, D., 2000. Diurnal cortisol rhythm as a predictor of breast cancer survival. Journal of the National Cancer Institute 92, 994-1000.

Silverman, M.N., Sternberg, E.M., 2012. Glucocorticoid regulation of inflammation and its functional correlates: from HPA axis to glucocorticoid receptor dysfunction. Annals of the New York Academy of Sciences 1261, 55-63.

Smyth, J.M., Ockenfels, M.C., Gorin, A.A., Catley, D., Porter, L.S., Kirschbaum, C., Hellhammer, D.H., Stone, A.A., 1997. Individual differences in the diurnal cycle of cortisol. Psychoneuroendocrinology $22,89-105$.

Smyth, N., Hucklebridge, F., Thorn, L., Evans, P., Clow, A., 2013. Salivary Cortisol as a Biomarker in Social Science Research. Social and Personality Psychology Compass 7, 605-625.

Solano, J.P., Gomes, B., Higginson, I.J., 2006. A comparison of symptom prevalence in far advanced cancer, AIDS, heart disease, chronic obstructive pulmonary disease and renal disease. Journal of pain and symptom management 31, 58-69. 
Stewart-Brown, S., Tennant, A., Tennant, R., Platt, S., Parkinson, J., Weich, S., 2009. Internal construct validity of the Warwick-Edinburgh Mental Well-being Scale (WEMWBS): a Rasch analysis using data from the Scottish Health Education Population Survey. Health and quality of life outcomes 7, 15-15.

Stone, A.A., Schwartz, J.E., Smyth, J., Kirschbaum, C., Cohen, S., Hellhammer, D., Grossman, S., 2001. Individual differences in the diurnal cycle of salivary free cortisol: a replication of flattened cycles for some individuals. Psychoneuroendocrinology 26, 295-306.

Thornton, L.M., Andersen, B.L., Blakely, W.P., 2010. The pain, depression, and fatigue symptom cluster in advanced breast cancer: covariation with the hypothalamic-pituitary-adrenal axis and the sympathetic nervous system. Health Psychology 29, 333-337.

Varadhan, R., Walston, J., Cappola, A.R., Carlson, M.C., Wand, G.S., Fried, L.P., 2008. Higher Levels and Blunted Diurnal Variation of Cortisol in Frail Older Women. Journal of Gerontology: Medical Sciences 63, 190-195.

von Leupoldt, A., Chan, P.-Y.S., Bradley, M.M., Lang, P.J., Davenport, P.W., 2011. The impact of anxiety on the neural processing of respiratory sensations. Neurolmage 55, 247-252.

von Leupoldt, A., Sommer, T., Kegat, S., Baumann, H.J., Klose, H., Dahme, B., Buchel, C., 2009. Dyspnea and pain share emotion-related brain network. Neurolmage 48, 200-206.

Weinrib, A.Z., Sephton, S.E., Degeest, K., Penedo, F., Bender, D., Zimmerman, B., Kirschbaum, C., Sood, A.K., Lubaroff, D.M., Lutgendorf, S.K., 2011. Diurnal Cortisol Dysregulation, Functional Disability, and Depression in Women with Ovarian Cancer. Cancer 116, 4410-4419.

Wijkstra, P.J., TenVergert, E.M., Van Altena, R., Otten, V., Postma, D.S., Kraan, J., Koeter, G.H., 1994. Reliability and validity of the chronic respiratory questionnaire (CRQ). Thorax 49, 465-467.

Woods, D.L., Kovach, C.R., Raff, H., Joosse, L., Basmadjian, A., Hegadoren, K.M., 2008. Using saliva to measure endogenous cortisol in nursing home residents with advanced dementia. Research in nursing \& health 31, 283-294.

Zigmond, A.S., Snaith, R.P., 1983. The Hospital Anxiety and Depression Scale. Acta Psychiatrica Scandinavica 67, 361-370. 
Appendix:

One-way between=subjects ANCOVA for diurnal cortisol slope by MRC Dyspnoea category (MRC $\leq 2$ versus $M R C \geq 3$ ), controlling for demographic, clinical and psychological covariates*

\begin{tabular}{|lllll|}
\hline Source & df & MS & F & p-value \\
\hline MRC category & 1 & 0.019 & 8.055 & .007 \\
Gender & 1 & 0.001 & 0.620 & .435 \\
Age & 1 & 0.000 & 0.111 & .740 \\
Socio-economic status & 1 & 0.000 & 0.068 & .795 \\
Diagnostic Category & 1 & $6.503 \mathrm{E}-7$ & 0.000 & .987 \\
Karnofsky Performance Status & 1 & $7.737 \mathrm{E}-5$ & 0.032 & .858 \\
Perceived Stress Scale Score & 1 & 0.000 & 0.159 & .692 \\
Breathlessness Intensity (NRS) & 1 & 0.001 & 0.494 & .486 \\
HADS-Anxiety & 1 & 0.001 & 0.490 & .487 \\
HADS-Depression & 1 & $7.361 \mathrm{E}-5$ & 0.031 & .862 \\
Intercept & 1 & 0.001 & 0.571 & .454 \\
Residual & 48 & 0.002 & & \\
\hline
\end{tabular}

*Note: $\mathrm{R}^{2}=0.274$ (adjusted $\mathrm{R}^{2}=0.123$ ), $\mathrm{p}=0.084$; Abbreviations: NRS, numerical rating scale; HADS-A, Hospital Anxiety and Depression Scale- Anxiety domain; HADS-D, Hospital Anxiety and Depression Scale- Depression domain. MRC, Medical Research Council 


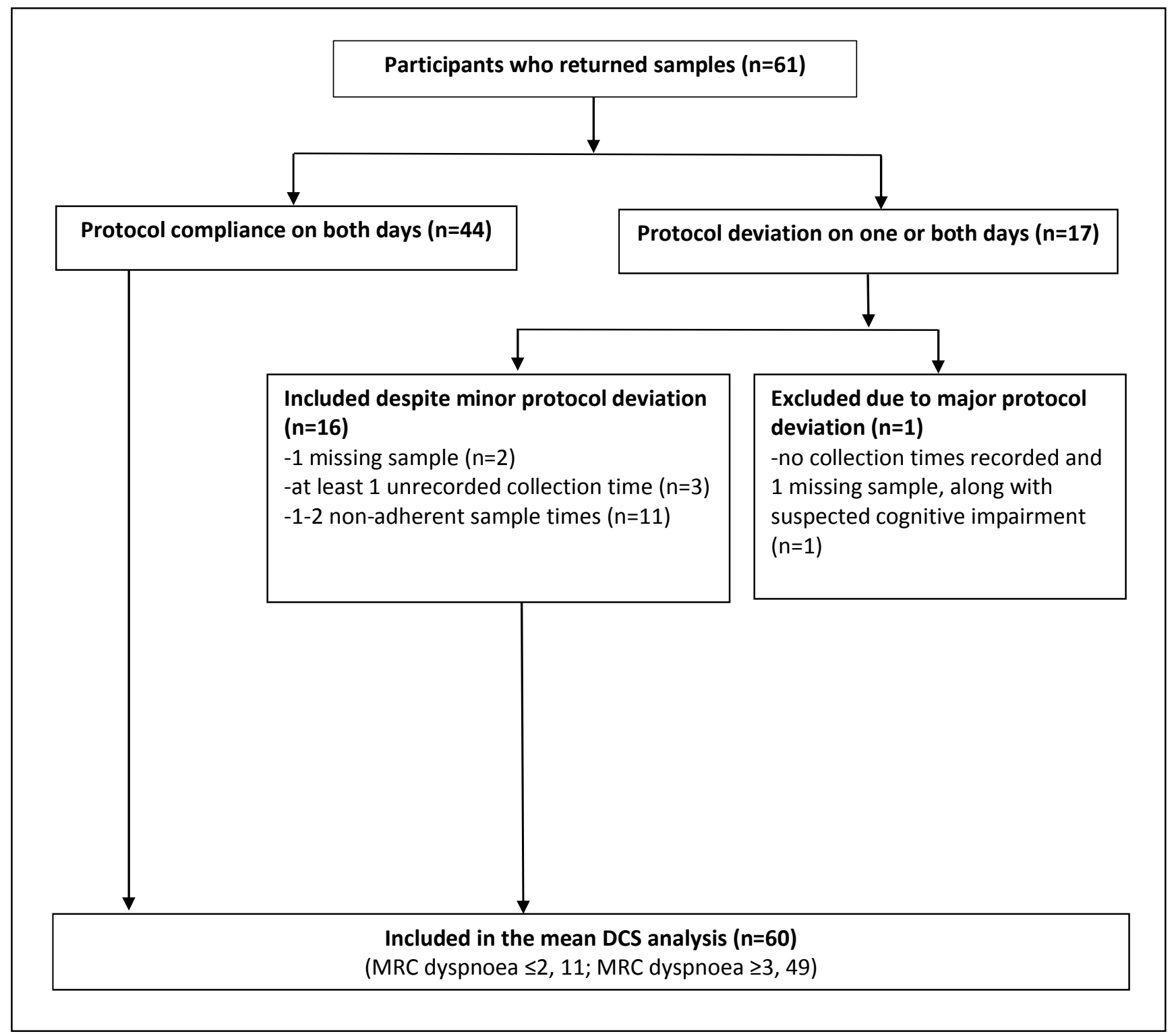

Figure 1. Summary of protocol compliance for the patient group. Abbreviations: DCS, diurnal cortisol slope; MRC, Medical Research Council 


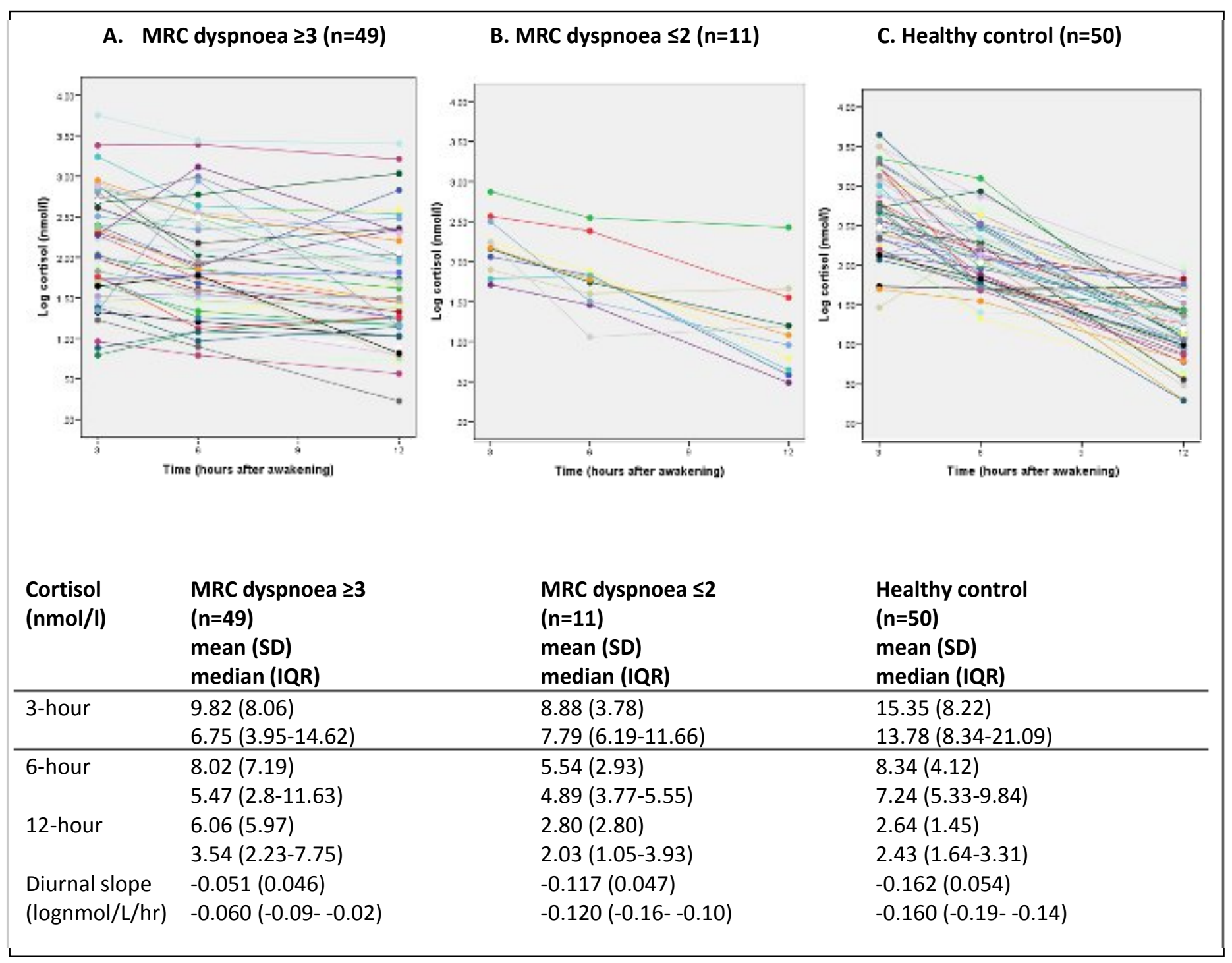

Figure 2. Top panel: log-transformed mean salivary cortisol profile over $\mathbf{2}$ days for each participant, with separate graphs $(\mathrm{A}-\mathrm{C})$ representing each group. Bottom panel: mean and median cortisol concentrations and diurnal slopes per group. 


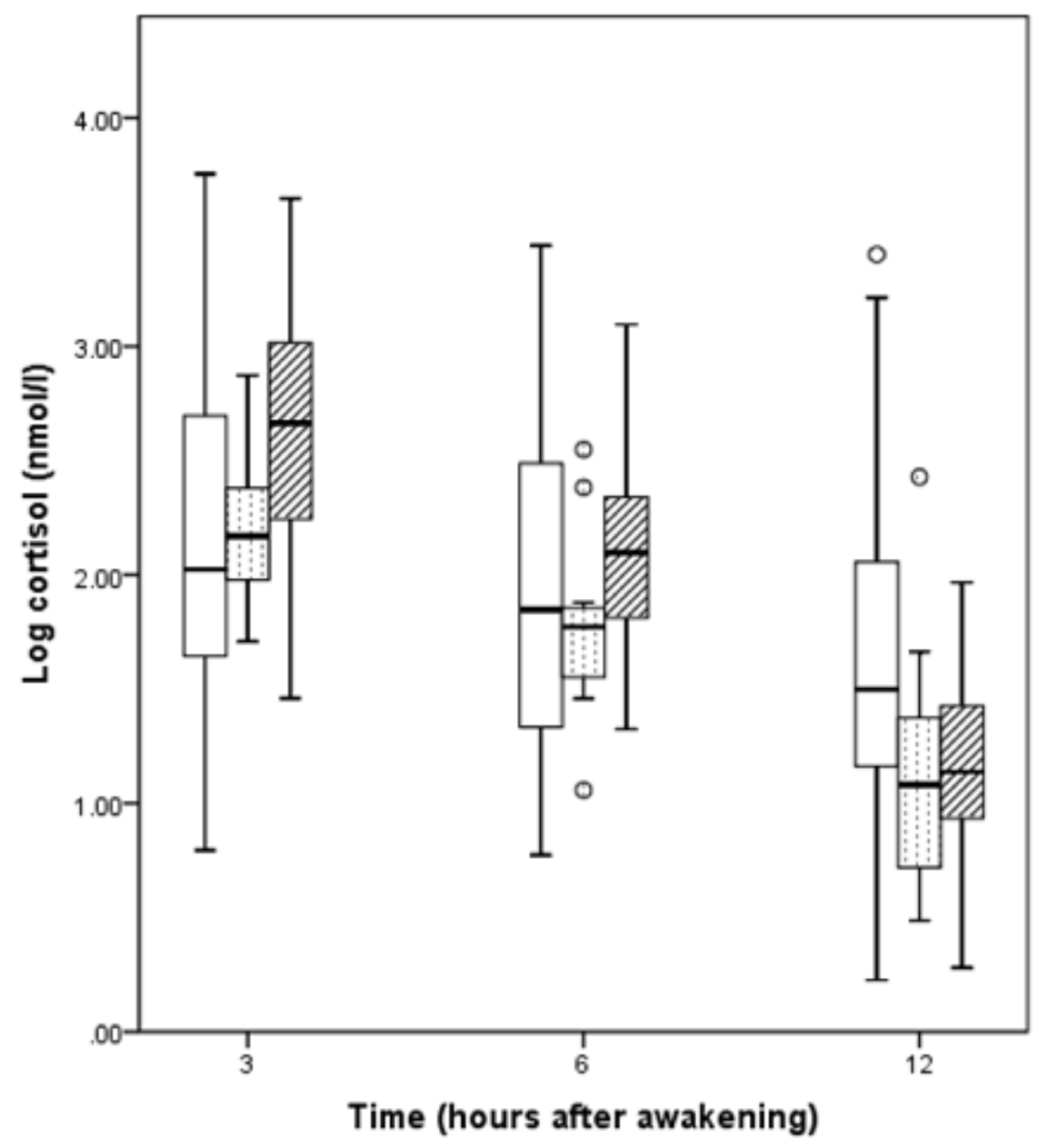

$\square$ MRC dyspnoea $\geq 3 \quad \square M R C$ dyspnoea $\leq 2 \quad$ \Healthy control

Figure 3. Boxplot showing the log-transformed cortisol values at 3, 6 and 12 hours after awakening for each study group. 


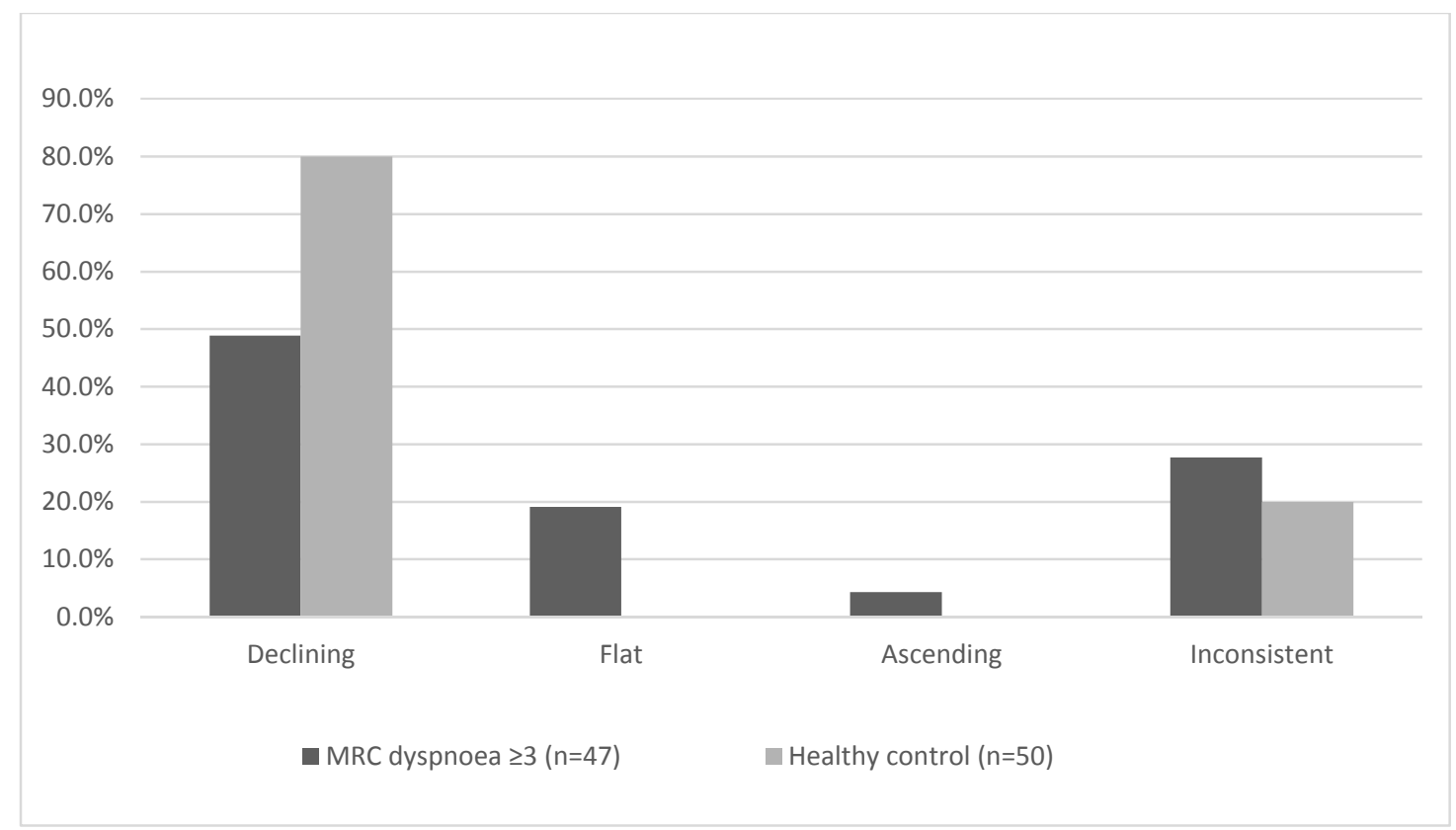

Figure 4. Frequency (\% per study group) of the four diurnal cycle categories in the 'MRC dyspnoea grade $\geq 3$ ' group versus the 'healthy control' group. Cycles were classified as 'inconsistent' if the diurnal slopes for day 1 and day 2 differed by $>0.08$. Cycles which were 'consistent' (day-difference $\leq 0.08$ ) fell into one of 3 other categories: 1 ) descending (slope $\leq-0.05), 2$ ) flat (slope>-0.05), or 3 ) ascending (slope $>0$ ). 